\title{
La investigación en instituciones de salud de mediana y alta complejidad del Valle de Aburrá, 20ll*
}

\section{Research in Mid and High Complexity Healthcare Facilities in the Aburra Valley, 20II}

\section{A pesquisa em instituições de saúde de mediana e alta complexidade do Valle de Aburrá, 20ll}

Fecha de recepción: 28-01-15 Fecha de aceptación: 18-03-15 Disponible en línea: 30-06-15 doi: 10.11144/Javeriana.rgyps18-28.liis

Como citar este artículo:

Arboleda-Posada GI, Cardona-Jiménez JL. La investigación en instituciones de salud de mediana y alta complejidad del Valle de Aburrá, 2011. Rev. Gerenc. Polít. Salud. 2015; 14(28): 97-108. http://dx.doi. org/10.11144/Javeriana.rgyps18-28.liis

\section{Gladys Irene Arboleda-Posada** \\ Jairo León Cardona-Jiménez***}

\footnotetext{
Artículo de investigación, como producto del proyecto Gestión del conocimiento en instituciones de salud, financiado por el Centro de Investigaciones, fondo de apoyo docente de la Facultad Nacional de Salud Pública de la Universidad de Antioquia, entre abril del 2011 y octubre del 2013, con código asignado INV-356-11. Esta publicación es el resultado de una investigación realizada con el apoyo financiero de la Estrategia de Sostenibilidad 2013-2014 del Comité para el Desarrollo de la Investigación (CODI) de la Universidad de Antioquia. Fue presentado en el III Congreso Internacional de Posgrados en Investigación de Administración y Estudios Organizacionales en la ciudad de Porto Alegre, Brasil. Un artículo resultado de la misma investigación se titula "La gestión del conocimiento y la investigación en instituciones de salud de mediana y alta complejidad del Valle de Aburrá 2011".

** Doctora en Administración, docente-investigadora, coordinadora del grupo de investigación Gestión y Políticas en Salud, Facultad Nacional de Salud Pública, Universidad de Antioquia, Medellín, Colombia. Correo electrónico: Gladys.arboleda@udea.edu.co - gladysarboledap@yahoo.com.co. Dirección postal: Universidad de Antioquia, Calle 62 No.52-59, Medellín, Colombia.

*** Magíster en Epidemiología. Docente investigador, coordinador del Grupo de Investigación en Salud del Adulto Mayor (Gisam), Corporación Universitaria Remington. Docente de cátedra de la Universidad de Antioquia. Correo electrónico: Jairo.cardona@remington.edu.co, Jairo.cardona@udea.edu.co.
} 


\section{Resumen}

Objetivo: identificar el nivel de conocimiento de los empleados acerca de las políticas de investigación en las instituciones de salud de mediana y alta complejidad en el Valle de Aburrá, Colombia. Metodología: investigación de corte transversal, con aplicación de encuestas a 224 empleados en las instituciones prestadoras de servicios salud de alta (52) y mediana complejidad (322), públicas y privadas en el 2011. Resultados: en referencia al conocimiento de la existencia de políticas de investigación, se encontró que este es mayor en los empleados de las instituciones de alta complejidad $(\mathrm{p}=0,000)$. Más del $70 \%$ de los empleados encuestados manifestaron su interés en trabajar en proyectos de investigación. Conclusión: las instituciones de salud de alta complejidad y públicas tuvieron más desarrollos en investigación y se percibió gran interés por la investigación en los empleados del sector salud, con independencia del nivel de complejidad, del tipo de institución y del nivel de formación.

Palabras clave: gestión del conocimiento; instituciones de salud; políticas; planificación; administración en salud; recursos para la investigación

\section{Abstract}

Objective: identifying the knowledge level of the employees regarding the research policies of mid and high complexity healthcare facilities in the Aburra Valley, Colombia. Methodology: cross-section research, with surveys for 224 employees of the healthcare providing institutions of high (52) and mid (322) complexity, both public and private, in 2011. Results: regarding the knowledge about the existence of research policies, we found that it is higher in the employees of high complexity institutions $(\mathrm{p}=0.000)$. Over $70 \%$ of the employees surveyed showed their interest in working on research projects. Conclusion: public high complexity healthcare facilities showed more developments on research and we found great interest of the employees of the health sector on research, regardless of the complexity level, the type of facility, and education level.

Keywords: knowledge management; healthcare facilities; policies; planning; health sector management; research resources

\section{Resumo}

Objetivo: identificar o nível de conhecimento dos empregados sobre as políticas de pesquisa nas instituições de saúde de mediana e alta complexidade no Valle de Aburrá, Colômbia. Metodologia: pesquisa de corte transversal, com aplicação de inquéritos a 224 empregados de instituições prestadoras de serviços de saúde de alta (52) e mediana complexidade (322), públicas e privadas no ano 2011. Resultados: no que diz respeito do conhecimento sobre a existência de políticas de pesquisa, encontrou-se que é maior em empregados de instituições de alta complexidade $(\mathrm{p}=0,000)$. Mais de $70 \%$ dos empregados indagados manifestaram interesse por trabalhar em projetos de pesquisa. Conclusão: as instituições de saúde de alta complexidade públicas tiveram maior desenvolvimento de pesquisa e percebeu-se grande interesse dos empregados do sector saúde pela pesquisa, com independência do nível de complexidade, do tipo de instituição e do nível de formação.

Palavras-chave: gestão do conhecimento; instituições de saúde; políticas; planejamento; administração em saúde; recursos para pesquisa 


\section{Introducción}

El conocimiento es la fuente del poder de más alta calidad y la clave para el cambio $(1,2)$; en todo el mundo se hace más ardua la batalla por controlar el conocimiento y los medios de comunicación. Un componente primordial en la generación de nuevo conocimiento es la investigación. Las instituciones de salud, por considerarse centros de conocimiento, ofrecen variadas opciones en investigación básica y aplicada.

Por investigación en salud se entiende "todo proceso dirigido a generar conocimiento sistemático y contrastar hipótesis dentro de las ciencias médicas, aunque no limitado a ellas, por cuanto se extiende además a las ciencias naturales y sociales" (3), en tanto que la innovación en salud está relacionada con la respuesta de las instituciones a los cambios del entorno y la adaptabilidad a las nuevas tecnologías (4). Las inversiones en investigación y desarrollo en salud favorecen la salud presente y futura de las poblaciones y generan un efecto positivo en el desarrollo económico y social de los países, al margen de su nivel de ingreso (3).

Un estudio diagnóstico de la situación de investigación en salud, realizado en Argentina en el año 2010, mostró que la mayoría de hospitales $(88 \%)$ realizaban actividades de gestión y difusión, relacionadas con la búsqueda de financiación, contactos con entidades científicas, evaluación de proyectos de los comités de ética, asesorías en investigación, participación en eventos científicos y publicación de artículos, mientras que el $56 \%$ estaba desarrollando el proyecto de investigación. Asimismo, el 61\% de las investigaciones contaron con financiación, el $41 \%$ de estas fueron financiadas por el sector privado (casas farmacéuticas) y el $43 \%$ por el sector público; el resto tuvieron una financiación de carácter mixto (5).
Uno de los temas más abordados en la investigación en salud en Colombia y otros países tiene relación con los recursos humanos (6). En Perú, esta agenda se propone como un eje articulador de iniciativas de investigación y canalización de los recursos públicos y privados y, entre otras funciones, la de construir redes que agilicen la gestión y faciliten el desarrollo de competencias investigativas en el personal de salud (7). A su vez, se plantean estrategias para su desarrollo (8).

En el artículo titulado "La unidad de investigación en hospitales auto gestionados", Seguel y Paravic (9) recomiendan considerar la investigación dentro de las estructuras organizacionales, con el propósito de mejorar la calidad en la atención, con participación del personal asistencial, a fin de innovar las prácticas y mejorar los estándares de calidad. Sin embargo, no se cuenta con políticas de investigación establecidas que incentiven a los centros asistenciales al desarrollo de propuestas de investigación. En Perú, por ejemplo, el $43 \%$ de las direcciones regionales de salud refirieron contar con una unidad de investigación, pero solo una de ellas era parte de la estructura orgánica (8).

Estudios nacionales e internacionales confirman que la investigación es un factor determinante en la gestión del conocimiento. En las instituciones de salud, la investigación clínica marca la dinámica hacia la generación de nuevo conocimiento. La publicación de las investigaciones en los hospitales, es más representativa en otros países de América Latina y el Caribe. De acuerdo al estudio realizado por Hernán Jaramillo (4), Colombia tenía una participación en investigación, del total de documentos en salud referenciados en la base de datos Thomson-Isi en el periodo 1999-2000, de 2,6\%, por debajo de países como Brasil (45,9\%), Argentina (19\%) y México (16\%), entre otros. 
En América Latina pocos países alcanzan a invertir el $1 \%$ del Producto Interno Bruto (PIB) en investigación en salud; el promedio latinoamericano es $0,54 \%$ (8), y Colombia no superó el 0,20\% (10) en el periodo 20062011. La Unión Europea (UE) aspiraba al 2010 dedicar el $3 \%$ del PIB a la investigación, con recursos procedentes tanto del sector público como del sector privado.

Eduardo Zarate (11) plantea estrategias para fortalecer la investigación con respecto al establecimiento de políticas en salud, mediante el impulso en el corto plazo a la innovación y a la tecnología, a través de la interdisciplinaridad; asimismo, vincular el quehacer del investigador con las necesidades nacionales y regionales, además de aplicar los resultados en la toma de decisiones, cumpliendo con la normas internacionales de ética científica y humana en investigación en salud. La investigación y las políticas en salud son procesos que deben complementarse. Las políticas deben resolver problemas y la investigación debe apoyar la toma decisiones (12).

En Colombia, la producción científica en medicina muestra la tendencia a la interdisciplinariedad, como fuente de desarrollo e integración de comunidades científicas. De acuerdo a los resultados obtenidos, el $69 \%$ de las investigaciones están a cargo de las universidades, a través de sus grupos de investigación; sin embargo, se debe destacar que los hospitales empiezan a desempeñar un papel importante como productores de conocimiento (4).

En Perú, según Miraval (13), hay pocos estudios sobre la producción y publicaciones científicas, y también escasa financiación y motivación para la investigación. En este sentido, recomienda consolidar esfuerzos entre formadores (instituciones académicas) y prestadores (instituciones de salud) que lleven al fortalecimiento de las líneas de investigación, creando evidencias científicas.
Según Jaramillo (4), las instituciones de salud como centros de investigación y conocimiento poseen un modelo organizacional capaz de proporcionar a sus médicos, clínicos y asociados, un espacio para el avance y el desarrollo de sus investigaciones; en ellos se integra la investigación, la innovación, la tecnología, la atención y la docencia. Para la creación de este conocimiento, se deben tener en cuenta variables como metas, objetivos, cultura organizacional, procesos normativos y procesos reglamentarios.

En el mismo trabajo (4), los resultados apuntan a que la relación con hospitales de alta calidad aumenta significativamente los niveles de éxito del profesional. Se encuentra que el inicio temprano de las actividades de investigación y docencia es significativo, lo cual sugiere su consolidación a lo largo de la carrera académica del investigador clínico, permitiéndole con ello alcanzar mayores niveles de éxito y mejores resultados en términos de publicación.

En las últimas décadas, el crecimiento de la información ha sido constante. Es por esta razón que la información y el conocimiento han sido establecidos como un recurso dentro de la institución que permite mejorar la productividady el desempeño, y es ahí donde cobra relevancia la investigación en temas relacionados con la medicina basada en evidencia e información científica, guías de práctica clínica, revisiones sistemáticas de la bibliografía y gestión del conocimiento, entre otros aspectos (14).

Sin embargo, se han encontrado barreras en la aplicación de los resultados de las investigaciones. Para algunos profesionales, estos resultados tienen gran significado e influencia en la práctica asistencial, pero no siempre son tenidos en cuenta para apoyar la toma de decisiones y los cambios estructurales en el interior de las instituciones (15). Martínez (16) habla de conflictos entre investigadores y tomadores de decisiones; un conflicto se 
refiere a la definición de prioridades, otro al manejo del tiempo y el lenguaje en que se emiten los resultados y la accesibilidad a estos.

Dada la importancia de la investigación en las instituciones de salud, como centros de innovación y conocimiento, esta investigación pretendió identificar el nivel de conocimiento de los empleados acerca de las políticas de investigación en las instituciones de salud de mediana y alta complejidad en el Valle de Aburrá, Colombia.

\section{Métodos}

Estudio de corte transversal. La información se recolectó mediante la aplicación de encuestas para la evaluación del conocimiento de las políticas de investigación. La población fue el personal que laboraba en las Instituciones Prestadores de Salud (IPS) de alta y mediana complejidad en el Valle de Aburrá en el año 2011, entendiendo IPS de alta complejidad como aquellas donde se atienden las diferentes especialidades médicas, incluyendo unidades especiales como cuidados intensivos y unidad renal, que corresponden al tercer nivel de atención. Las IPS de mediana complejidad corresponden a instituciones que cuentan con atención de las especialidades básicas como pediatría, cirugía general, medicina interna, ortopedia y ginecobstetricia. La población de IPS fueron 374 , de las cuales 52 son de complejidad alta y 322 de complejidad media. Para llegar al número de empleados por encuestar, se solicitó por vía telefónica información sobre la cantidad aproximada de empleados a las 69 instituciones elegidas aleatoriamente. El total de la muestra fue de 224 empleados.

Los criterios de inclusión fueron: personal que laboraba en IPS de mediana y alta complejidad ubicadas en el Valle de Aburrá al momento de aplicación de la encuesta, con más de un año de laborar en la institución, sin importar el tipo de contrato, con formación técnica, tecnológica, profesional, especialista, magíster o doctorado. Se excluyeron los empleados con cargos directivos de primero y segundo rango, de acuerdo a cada estructura orgánica.

El instrumento fue aplicado por el investigador principal y dos estudiantes de último semestre del programa de Administración en Salud, previamente capacitados, con compresión de la terminología empleada y habilitados para explicar las dudas de los encuestados. El instrumento se aplicó de manera individual y personal.

El contenido de las preguntas fue desarrollado a partir de varios trabajos realizados acerca de temáticas relacionadas (17-19). Con el fin de verificar la eficacia de la encuesta, se realizó la prueba piloto en una IPS de alta complejidad, que corroboró su validez. El tipo de preguntas fue estructurado.

En el análisis se buscó la asociación entre políticas de investigación, fuentes de financiación, relación con universidades (convenios docentes asistenciales o de cooperación cientifica), interés en la investigación, existencia de asociación de expertos, participación en proyectos, incentivos por compartir el conocimiento según el tipo de entidad, grado de complejidad de las IPs y formación académica. Mediante pruebas Chi cuadrado y proporciones, en todos los casos, se consideró como significativo cualquier valor-p menor de 0,05 .

La información recibida fue de carácter confidencial, se utilizó únicamente para fines académicos y para dar cumplimiento a los objetivos propuestos. Asimismo, se garantizó la omisión de los nombres de los encuestados. Las preguntas hechas no tocaron la intimidad. Además, se contó con el consentimiento informado, según lo establece la Resolución 8430 de 1993 del Ministerio de Salud de Colombia (20). 


\section{Resultados}

El personal encuestado fue en gran proporción del sexo femenino ( $80 \%$ ), el mayor porcentaje se encontró en el rango de edad entre 30 y 49 años (57\%), una tercera parte de la población fue menor de 30 años. El $54 \%$ fueron técnicos o tecnólogos, $31 \%$ profesionales y $15 \%$ con posgrado. El $66 \%$ se dedicaban a actividades asistenciales y $34 \%$ a labores administrativas.

De los encuestados, $82(37 \%)$ respondieron afirmativamente a la pregunta: ¿La Institución tiene definidas políticas de investigación e innovación? De estos, solo 12 expresaron conocer algunas, como: seguridad del paciente, adquisición de nuevos equipos, uso de tecnología de punta, mejoramiento del programa de calidad de la atención en salud y convenios para el desarrollo de la investigación con otras entidades y universidades (ver figura 1).

La existencia de políticas de investigación e innovación no depende del tipo de entidad, una tercera parte respondió afirmativamente, otra no, y el resto manifestó no saber. Analizando desde el nivel de complejidad, se encontró que tienen mayor conocimiento con relación al tema los empleados de las instituciones de alta complejidad.

Hay asociación entre la formación académica y el conocimiento sobre la existencia de políticas de investigación e innovación. El 44,2\% de los que tenían menor nivel de formación académica (técnico o tecnólogo) sostuvieron que la institución cuenta con estas políticas; sin embargo, los profesionales y los especialistas opinaron en igual proporción que estas no existen. Igualmente, casi la tercera parte de los empleados desconocían si existían estas políticas de investigación en la institución donde laboran (ver tabla 1).

Hay relación positiva entre los empleados que opinan que en sus instituciones tienen definidas políticas de investigación e innovación y aquellos que manifiestan que se cuenta con fuentes de financiación para estas actividades. El 83\% de

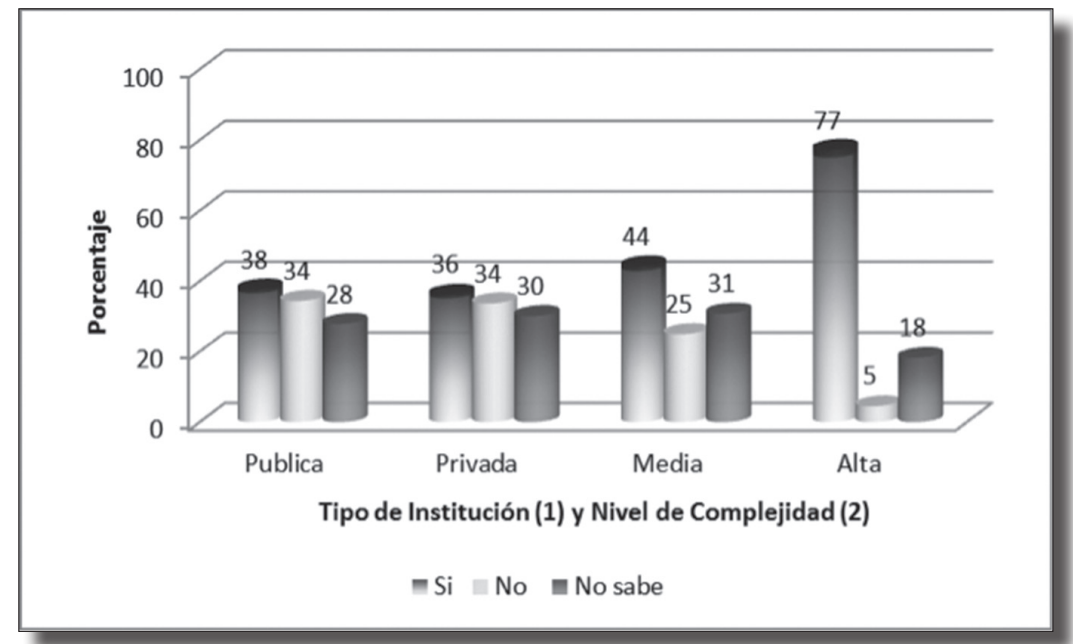

Figura 1. Políticas de investigación e innovación Según tiPo de institución y NiVel de Complejidad

(1) $\mathrm{X}^{2}=0,106, \mathrm{gl}=2, \mathrm{p}=0,948,0$ casillas $(0,0 \%)$ tienen una frecuencia esperada inferior a 5, son independientes.

(2) $\mathrm{X}^{2}=41,034, \mathrm{gl}=2, \mathrm{p}=0,000,0$ casillas $(0,0 \%)$ tienen una frecuencia esperada inferior a 5, son dependientes. Fuente: elaboración propia 


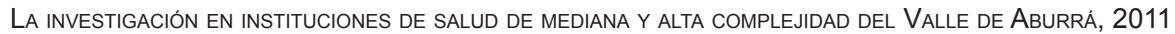

Tabla 1. Políticas de investigación e innovación SEgún formación aCAdÉmica

\begin{tabular}{|c|c|c|c|c|c|c|c|c|}
\hline \multirow{3}{*}{$\begin{array}{c}\text { Políticas de investigación } \\
\text { e innovación }\end{array}$} & \multicolumn{8}{|c|}{ Formación académica } \\
\hline & \multicolumn{2}{|c|}{ Técnico o tecnólogo } & \multicolumn{2}{|c|}{ Profesional } & \multicolumn{2}{|c|}{ Especialista } & \multicolumn{2}{|c|}{ Total } \\
\hline & $\mathbf{n}$ & $\%$ & $\mathbf{n}$ & $\%$ & $\mathbf{n}$ & $\%$ & $\mathbf{n}$ & $\%$ \\
\hline Sí & 53 & 44,2 & 16 & 22,9 & 13 & 38,2 & 82 & 36,6 \\
\hline No & 30 & 25,0 & 31 & 44,3 & 15 & 44,1 & 76 & 33,9 \\
\hline No sabe & 37 & 30,8 & 23 & 32,9 & 6 & 17,6 & 66 & 29,5 \\
\hline Total & 120 & 100,0 & 70 & 100,0 & 34 & 100,0 & 224 & 100,0 \\
\hline
\end{tabular}

$\mathrm{X}^{2}=13,547, \mathrm{gl}=4, \mathrm{p}=0,009,0$ casillas $(0,0 \%)$ tienen una frecuencia esperada inferior a 5 , son dependientes.

Fuente: elaboración propia

las instituciones que contaban con políticas definidas, a su vez tenían fuentes de financiación para estas. De manera opuesta, las instituciones que no contaban con estas políticas, tampoco disponían de fuentes de financiación, en un $76 \%$. Esta asociación es estadísticamente significativa (resultados no mostrados).

El 39\% de los encuestados de las instituciones privadas expresaron que estas no cuentan con fuentes de financiación para investigación e innovación; lo mismo sostuvo el $34 \%$ en las entidades públicas. Igualmente, más de la tercera parte expresó no saberlo, con independencia del tipo de institución (ver figura 2).

Hay dependencia entre las variables de fuentes de financiación y complejidad. Se destacó que $71 \%$ de los empleados de las instituciones de alta complejidad señalaron que estas cuentan con fuentes de financiación, a diferencia de las de complejidad media, en donde fue de este parecer el $16 \%$ de los encuestados.

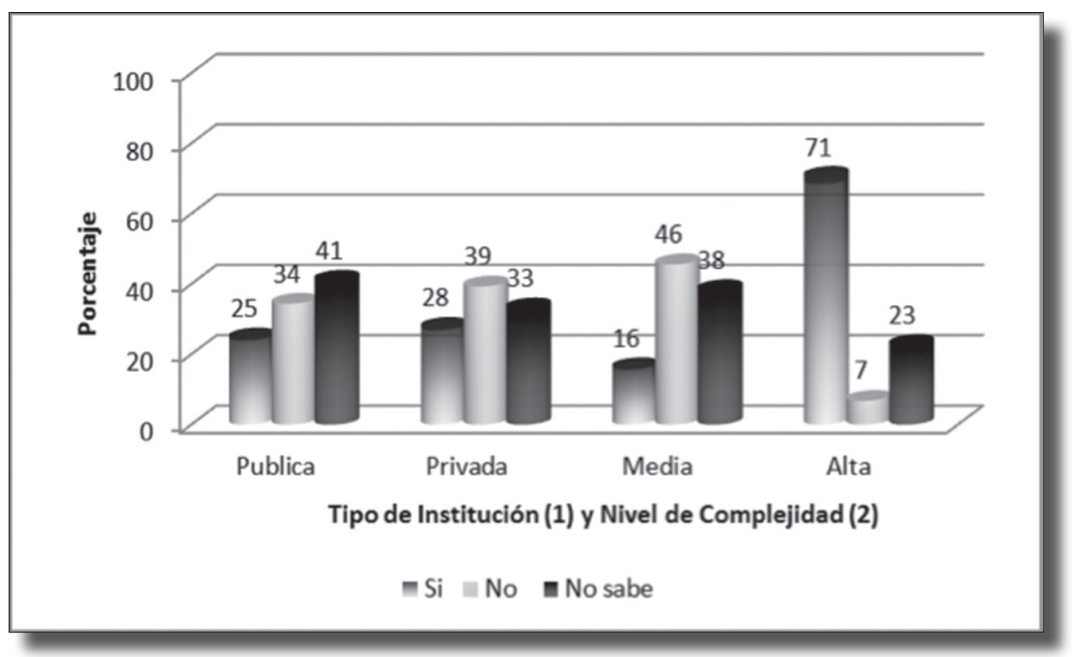

FiguRa 2. FuENTES DE FINANCIACIÓN PARA LA INVESTIGACIÓN Y LA INNOVACIÓN SEGÚN TIPO DE INSTITUCIÓN Y NIVEL DE COMPLEJIDAD

(1) $\mathrm{X}^{2}=1,201, \mathrm{gl}=2, \mathrm{p}=0,548,0$ casillas $(0,0 \%)$ tienen una frecuencia esperada inferior a 5, son independientes.

(2) $\mathrm{X}^{2}=55,406, \mathrm{gl}=2, \mathrm{p}=0,000,0$ casillas $(0,0 \%)$ tienen una frecuencia esperada inferior a 5 , son dependientes. Fuente: elaboración propia 
Los empleados de cargos administrativos fueron más conocedores de la existencia de fuentes de financiación en la institución (35\%), si se compara con los asistenciales (22\%). Se destacó que el $39 \%$ del personal asistencial y el $29 \%$ de los administrativos desconocían si la institución contaba o no con fuentes de financiación para la investigación y la innovación (resultados no mostrados).

De la tabla 2 se concluye que el 35,6\% de las instituciones que tenían fuentes de financiación para la investigación y la innovación, a su vez manifestaron mantener relaciones con universidades y centros de formación; en sentido opuesto, el $52,6 \%$ de las que no contaban con esta financiación tampoco manifestaron tener relación con las instituciones académicas (ver tabla 2).

En el mismo sentido, se muestra la relación entre la existencia de fuentes de financiación para la investigación y la innovación y la existencia de asociaciones de expertos con intereses comunes. Igualmente, esta misma relación se da con la participación en proyec-

Tabla 2. Fuentes de FinANCIACión PARA LA INVESTIGACIÓN Y LA INNOVACIÓN SEGÚN RELACIÓN CON UNIVERSIDADES, ASOCIACIÓN DE EXPERTOS, PARTICIPACIÓN EN PROYECTOS E INCENTIVOS POR COMPARTIR CONOCIMIENTOS

\begin{tabular}{|c|c|c|c|c|c|c|}
\hline \multirow{3}{*}{$\begin{array}{l}\text { Fuentes de financiación } \\
\text { para la investigación y la } \\
\text { innovación }\end{array}$} & \multicolumn{6}{|c|}{ Relación con universidades (1) } \\
\hline & \multicolumn{2}{|c|}{ Sí } & \multicolumn{2}{|c|}{ No } & \multicolumn{2}{|c|}{ Total } \\
\hline & $\mathbf{n}$ & $\%$ & $\mathbf{n}$ & $\%$ & $\mathbf{n}$ & $\%$ \\
\hline Sí & 52 & 35,6 & 8 & 10,3 & 60 & 26,8 \\
\hline No & 44 & 30,1 & 41 & 52,6 & 85 & 37,9 \\
\hline No sabe & 50 & 34,2 & 29 & 37,2 & 79 & 35,3 \\
\hline Total & 146 & 100,0 & 78 & 100,0 & 224 & 100,0 \\
\hline \multicolumn{7}{|c|}{ Asociación de expertos (2) } \\
\hline Sí & 34 & 59,6 & 26 & 15,6 & 60 & 26,8 \\
\hline No & 12 & 21,1 & 73 & 43,7 & 85 & 37,9 \\
\hline No sabe & 11 & 19,3 & 68 & $4 p, 7$ & 79 & 35,3 \\
\hline Total & 57 & 100,0 & 167 & 100,0 & 224 & 100,0 \\
\hline \multicolumn{7}{|c|}{ Participación en proyectos (3) } \\
\hline Sí & 35 & 37,6 & 25 & 19,1 & 60 & 26,8 \\
\hline No & 34 & 36,6 & 51 & 38,9 & 85 & 37,9 \\
\hline No sabe & 24 & 25,8 & 55 & 42,0 & 79 & 35,3 \\
\hline Total & 93 & 100,0 & 131 & 100,0 & 224 & 100,0 \\
\hline \multicolumn{7}{|c|}{ Incentivos por compartir conocimientos (4) } \\
\hline Sí & 28 & 39,4 & 32 & 20,9 & 60 & 26,8 \\
\hline No & 23 & 32,4 & 62 & 40,5 & 85 & 37,9 \\
\hline No sabe & 20 & 28,2 & 59 & 38,6 & 79 & 35,3 \\
\hline Total & 71 & 100,0 & 153 & 100,0 & 224 & 100,0 \\
\hline
\end{tabular}

(1) $\mathrm{X}^{2}=19,069, \mathrm{gl}=2, \mathrm{p}=0,000,0$ casillas $(0,0 \%)$ tienen una frecuencia esperada inferior a 5 , son independientes (2) $\mathrm{X}^{2}=42,106, \mathrm{gl}=2, \mathrm{p}=0,000,0$ casillas $(0,0 \%)$ tienen una frecuencia esperada inferior a 5 , son dependientes. (3) $\mathrm{X}^{2}=8,541, \mathrm{gl}=2, \mathrm{p}=0,014,0$ casillas $(0,0 \%)$, tienen una frecuencia esperada inferior a 5, son dependientes. (4) $\mathrm{X}^{2}=8,541, \mathrm{gl}=2, \mathrm{p}=0,014,0$ casillas $(0,0 \%)$ tienen una frecuencia esperada inferior a 5 , son dependientes. Fuente: elaboración propia 
tos e incentivos por compartir conocimientos. Estas diferencias fueron significativas.

El 96\% de los empleados de las instituciones de alta complejidad reportaron mantener relaciones académicas con universidades y centros de formación especializada, en comparación con el 58\% en las de mediana complejidad. A su vez, las instituciones públicas reportaron en un $97 \%$ que tenían esta relación, en contraste con el 53\% de las privadas. Estas asociaciones fueron significativas (resultados no mostrados).

En las instituciones que mantenían relaciones académicas con universidades y centros de formación especializada, se halló más personal interesado en participar en proyectos de investigación. De la misma manera, el mayor número del personal que había participado en grupos para desarrollar proyectos, había recibido estímulos para la asistencia a seminarios, congresos, diplomados u otros cursos de capacitación o actualización. Asimismo, una mayor proporción de empleados se habían visto beneficiados por programas institucionales que propician su desarrollo profesional, en comparación con los empleados de aquellas instituciones que no poseían este tipo de relaciones con las universidades. Las anteriores asociaciones fueron significativas (ver tabla 3 ).

TABLA 3. ReLACIÓN CON UNIVERSIDADES SEGÚN INTERÉS EN LA INVESTIGACIÓN

\begin{tabular}{|c|c|c|c|c|c|c|}
\hline \multirow{3}{*}{$\begin{array}{l}\text { Relación con } \\
\text { universidades }\end{array}$} & \multicolumn{6}{|c|}{ Interés en la investigación (1) } \\
\hline & \multicolumn{2}{|c|}{ Sí } & \multicolumn{2}{|c|}{ No } & \multicolumn{2}{|c|}{ Total } \\
\hline & $\mathbf{n}$ & $\%$ & $\mathbf{n}$ & $\%$ & $\mathbf{n}$ & $\%$ \\
\hline Sí & 118 & 70,7 & 28 & 49,1 & 146 & 65,2 \\
\hline No & 49 & 29,3 & 29 & 50,9 & 78 & 34,8 \\
\hline Total & 167 & 100,0 & 57 & 100,0 & 224 & 100,0 \\
\hline \multicolumn{7}{|c|}{ Participación en proyectos (2) } \\
\hline Sí & 71 & 76,3 & 75 & 57,3 & 146 & 65,2 \\
\hline No & 22 & 23,7 & 56 & 42,7 & 78 & 34,8 \\
\hline Total & 93 & 100,0 & 131 & 100,0 & 224 & 100,0 \\
\hline \multicolumn{7}{|c|}{ Estímulos a la educación complementaria (3) } \\
\hline Sí & 89 & 77,4 & 57 & 52,3 & 146 & 65,2 \\
\hline No & 26 & 22,6 & 52 & 47,7 & 78 & 34,8 \\
\hline Total & 115 & 100,0 & 109 & 100,0 & 224 & 100,0 \\
\hline \multicolumn{7}{|c|}{ Beneficiados de programas de desarrollo profesional (4) } \\
\hline Sí & 83 & 79,0 & 63 & 52,9 & 146 & 65,2 \\
\hline No & 22 & 21,0 & 56 & 47,1 & 78 & 34,8 \\
\hline Total & 105 & 100,0 & 119 & 100,0 & 224 & 100,0 \\
\hline
\end{tabular}

(1) $\mathrm{X}^{2}=7,761, \mathrm{gl}=1, \mathrm{p}=0,0050$ casillas $(0,0 \%)$ tienen una frecuencia esperada inferior a 5, son dependientes.

(2) $\mathrm{X}^{2}=7,914, \mathrm{gl}=1, \mathrm{p}=0,0030$ casillas $(0,0 \%)$ tienen una frecuencia esperada inferior a 5 , son dependientes

(3) $\mathrm{X}^{2}=15,531, \mathrm{gl}=1, \mathrm{p}=0,0000$ casillas $(0,0 \%)$ tienen una frecuencia esperada inferior a 5, son dependientes (4) $\mathrm{X}^{2}=16,751, \mathrm{gl}=1, \mathrm{p}=0,0000$ casillas $(0,0 \%)$ tienen una frecuencia esperada inferior a 5, son dependientes. Fuente: elaboración propia 




Figura 3. INTERÉS EN LA INVESTIGACIÓN SEGÚN TIPO DE INSTITUCIÓN Y NIVEL DE COMPLEJIDAD

(1) $\mathrm{X}^{2}=1,085, \mathrm{gl}=1, \mathrm{p}=0,298,0$ casillas $(0,0 \%)$ tienen una frecuencia esperada inferior a 5 , son independientes.

(2) $\mathrm{X}^{2}=1,084, \mathrm{gl}=1, \mathrm{p}=0,2980,0$ casillas $(0,0 \%)$ tienen una frecuencia esperada inferior a 5 , son independientes. Fuente: elaboración propia

El $80 \%$ de los empelados encuestados de instituciones públicas y el $72 \%$ en las instituciones privadas manifestaron su interés en trabajar en proyectos de investigación, siendo esto un poco más representativo en los encuestados del sector público. En cuanto al nivel de complejidad, tanto en las instituciones de nivel medio como en las de nivel alto, los encuestados se expresaron en forma positiva, en porcentajes similares al de tipo de institución, siendo más notable el intereses entre los miembros de instituciones de alta complejidad (ver figura 3).

\section{Discusión}

$\mathrm{Al}$ revisar la literatura, no se hallaron estudios en la propia temática que permitieran realizar análisis comparativos, por lo cual la discusión se hará con base en las generalidades encontradas. conocimiento de la existencia de políticas de investigación e innovación, pero, en general, el personal no tiene claridad de la existencia de tales políticas. El $63 \%$ manifestó que estas políticas no existían o desconocía su existencia. Zarate (11) plantea que se deben definir estrategias que permitan un fortalecimiento de la investigación, mediante la implementación de prácticas que sintonicen al investigador con el medio interdisciplinar, tanto a nivel nacional como a nivel internacional, con el fin de introducir los resultados de las investigaciones en el desarrollo institucional, a través de la toma de decisiones (12).

Asimismo, el $73 \%$ de los encuestados no saben o no conocen si la institución tiene fuentes de financiación para la investigación y la innovación; además, manifestaron que las instituciones no cuentan con recursos suficientes para su ejecución, lo cual se hace en alianza con las universidades o en cofinanciación con las casas farmacéuticas. El que haya poca investigación en instituciones de salud en Colombia concuerda con lo manifestado por Barragán (10), quien señala que 
en el país la inversión en investigación como porcentaje del PIB no superó el 0,20\% en el periodo 2006-2011, cifra que resulta muy baja, comparada con América Latina y el mundo, donde este índice supera el $0,5 \%$ en países en vía de desarrollo y entre 2 y $3,5 \%$ en países industrializados (21). En el mismo sentido, la Organización Panamericana de la Salud (OPS) (22) afirma: "La investigación sanitaria no debe ser dominio exclusivo de las instituciones académicas sino que debe incluir a los prestadores de salud, los tomadores de decisiones y la sociedad civil"; además, se deben dedicar recursos para cerrar las brechas de conocimiento relacionadas con las prioridades sanitarias y cuyos resultados se traduzcan en "mensajes para la toma de acciones".

De acuerdo a los resultados obtenidos en la investigación realizada en el 2007 por el Ministerio de la Protección Social y el Centro para el Desarrollo y Evaluación de Políticas y Tecnología en Salud Pública de la Universidad del Valle (23), "la mayoría de los grupos de investigación en ciencias básicas se encuentran en las universidad públicas $(53,27 \%)$, en tanto que la investigación básica es casi inexistente en las instituciones Prestadoras de Servicios de Salud, tanto públicas como privadas". Sin embargo, Jaramillo et al. (4) encontraron que de la producción de artículos colombianos en salud entre 1975 y 2005, 14,8\% fueron realizados en clínicas y hospitales. El hospital, considerado un centro de investigación y conocimiento, alberga la infraestructura capaz de proporcionar tanto al área clínica como al área administrativa un espacio para el avance y desarrollo de sus investigaciones. Le corresponde asimismo hacer el cambio a una nueva cultura organizacional: "la organización del conocimiento".

También se evidenció que un alto porcentaje de los encuestados estaban interesados en participar en algún proyecto de investigación, espe- cialmente en instituciones de alta complejidad públicas, así como en aquellas que mantienen una relación cercana con los centros de formación y universidades, las cuales, según Jaramillo (4) son pioneras en el país en investigación. Las instituciones deben aprovechar este interés del personal para participar en investigación, estimulando la formación y la capacitación, para la mejor utilización del conocimiento, tal como lo recomienda la ops (22).

En síntesis, las instituciones de salud de alta complejidad y públicas tienen más desarrollos en investigación; no obstante, gran parte de su personal desconoce la existencia de estas políticas. Un número importante de empleados manifiestan que en las instituciones no existen recursos financieros destinados a la investigación, o dicen desconocer si tales recursos existen o no, aunque en algunos casos, especialmente en instituciones de alta complejidad, se puede disponer de recursos y cofinanciación de entidades externas para el desarrollo de las investigaciones. Se percibe, de igual manera, gran interés por la investigación entre los empleados del sector salud, con independencia del nivel de complejidad, del tipo de institución y del nivel de formación.

Se recomienda que las instituciones de salud, en particular las de mediana complejidad, incluyan en sus programas políticas de investigación que permitan destinar recursos financieros para el desarrollo de estas y trabajar conjuntamente con instituciones académicas y laboratorios que apoyen ampliamente la investigación; además, establecer alianzas con las universidades para fortalecer la investigación, especialmente en el caso de las instituciones de mediana complejidad, donde se percibió un número limitado de contactos con estas instituciones; por último, se deben elaborar programas de capacitación y formación para el personal interesado en la investigación. 


\section{Referencias bibliográficas}

1. Toffler A. Avances y premisas. Barcelona: Plaza \& Janés; 1983.

2. Incolda Mundo Gerencial. La gerencia en los 95 años de Peter Drucker [Internet] [acceso: 13 de septiembre del 2013]. Disponible en: http:// es.scribd.com/doc/126318565/Mundo-GerencialPeter-Drucker.

3. Maceira D, Paraje G, Aramayo F, Duarte Masi S, Sánchez D. Financiamiento público de la investigación en salud en cinco países de América Latina. Rev Panam Salud Pública. 2010 Jun.; 27 (6): 442-51.

4. Jaramillo H, Latorre C, Lopera C, Albán C. El hospital como organización de conocimiento y espacio de investigación y formación [Internet] [acceso: 30 de mayo del 2012]. Disponible en: http:// www.consultorsalud.com/biblioteca/documentos/ hospital_conocimiento_investigacion.pdf

5. Bonet F, Allegri R, Bernet J, Bossio P, Clacheo R, Hijano D et al. Diagnóstico de situación de la investigación en salud en el ámbito del ministerio de salud de la nación y 10 ministerios provinciales. Rev Argent Salud Pública. 2012; 3 (10): 6-14.

6. Ministerio de la Protección Social. Proyecto Plan de largo plazo para el desarrollo y fortalecimiento de los recursos humanos de salud en Colombia. Los recursos humanos de la salud en Colombia. Balance, competencias y prospectiva [Internet] [acceso: 3 de junio del 2014]. Disponible en: http://www.minsalud.gov.co/salud/Documents/ LOS \%20RECURSOS \%20HUMANOS \%20DE \%20LA $\% 20$ SALUD \%20EN\%20COLOMBIA.pdf

7. Yagui M, Vargas J. La agenda nacional de investigación en recursos humanos en salud: generando evidencias para mejorar las competencias en el sector salud. Rev Peru Med Exp Salud Pública. 2011; 28 (2): 175-6.

8. Yagui M, Espinoza M, Caballero P, Castilla T, Garro $\mathrm{G}$ et al. Avances y retos en la construcción del sistema nacional de investigación en salud en el Perú. Rev Perú Med Exp Salud Pública. 2010 sept; 27: 387-97.

9. Seguel F, Paravic T. Unidad de investigación en hospitales auto gestionados. Ciencia y Enfermería. 2011; 17 (2): 19-25.

10. Barragán J. ¿Pena de muerte a la investigación en Colombia? [Internet] [acceso: 8 de agosto del 2012]. Disponible en: http://comunidad.semana. com/t5/A-pupitrazo-limpio/Pena-de-muerte-a-lainvestigaci\%C3\%B3n-en-Colombia/ba-p/19339

11. Zarate E. Innovación en la investigación. Simposio: Políticas de investigación en salud. Rev Peru Med Exp Salud Pública. 2010; 27 (3): 432-7.

12. Gómez R, Orozco D, Rodríguez F, Velásquez W. Políticas públicas y salud: relación entre investigación y decisión. Rev Fac Nac Salud Pública. 2006 Jul-Dic; 24 (2): 105-18.

13. Miraval E. Investigación académica y de los recursos humanos en Salud. Acta Med Per. 2012; 29 (4): 213-5.
14. Macías Chapula CA. La gestión del conocimiento en el área de la salud. Rev Eviden Invest Clin. 2009; 2 (1): 31-5.

15. Brito Rocha E, Nagliate P, Bis Furlan C, Rocha Jr K, Trevizan MA, Mendes Costa I. Gestión del conocimiento en salud: revisión sistemática de la literatura. Rev Latino-Am Enfermagem. 2012 Abr. 20 (2): 392-400 [Internet] [acceso: 8 de agosto del 2012]; Disponible en: http://www.revistas.usp.br/ rlae/article/viewFile/48520/52427

16. Martínez N. Revisión en la aplicación de los resultados de la investigación en sistemas y servicios de salud por los profesionales de enfermería. Horiz Enferm. 2012; 23 (3): 23-31.

17. Arboleda Posada GI. Desarrollo humano en el personal vinculado a las instituciones de tercer nivel de atención: Servicios de salud y bienestar laboral Medellín, Colombia, 2007. Vis Futuro. 2011; 15 (1) [Internet] [acceso: 18 de marzo del 2015]. Disponible en: <http://www.scielo.org. ar/scielo.php? script $=$ sci_arttext $\&$ pid $=$ S 1668 $87082011000100005 \& \operatorname{lng}=\bar{e} \& n r m=$ iso $>$.

18. Arboleda GI. Ingreso y capacitación del personal vinculado a instituciones del tercer nivel de atención en salud. Medellín, Colombia. Rev Gerenc Polit Salud. 2012 Jul; 11 (23): 134-51.

19. Arboleda GI. Percepción que los gerentes tienen acerca de la gestión del conocimiento en las instituciones de salud, Valle de Aburrá, 2011. Rev Fac Nac Salud Pública. 2014 mayo; 32 (2): 40- 51.

20. República de Colombia. Ministerio de Salud. Resolución Número 8430 de octubre 4 de 1993 por la cual se establecen las normas científicas, técnicas y administrativas para la investigación en salud. Bogotá: El Ministerio; 1993.

21. El Tiempo. América Latina con pocas universidades de investigación [Internet] [acceso: 8 de agosto del 2012]. Disponible en: http://www.eltiempo.com/ vida-de-hoy/ciencia/ARTICULO-WEB-NEW_NOTA_INTERIOR-12121808.html

22. Porrás A. Organización Panamericana de la Salud (ops). La investigación en salud como instrumento para mejorar la salud pública. Proyecto de Promoción y Desarrollo de la Salud. Área de Tecnología, Atención e Investigación Sanitaria [Internet]. [acceso: 8 de agosto del 2012]. Disponible en: http:// www.eventos.bvsalud.org/agendas/Bvs-COR/public/ documents/analia_porras-154419.pdf

23. Centro de Investigaciones de la Universidad del Valle (Cedetes), Centro colaborador de la Organización Mundial de la Salud, Ministerio de la Protección Social. Lineamientos para una política nacional de investigación en salud. En: Plan Estratégico 2009- 2015. Programa Nacional de Ciencia y Tecnología de la Salud. Bogotá: Colciencias; 2008. pp. 26-27.

Agradecimientos: esta publicación es el resultado de una investigación realizada con el apoyo financiero de la Estrategia de Sostenibilidad 2013-2014, del Comité para el Desarrollo de la investigación (CODI), Universidad de Antioquia. 